\title{
SOME POSSIBLE IMPLICATIONS OF THE RADIO EMISSION OF SCO X-1
}

\author{
P. A. FELDMAN and J. I. SILK \\ Institute of Theoretical Astronomy, Cambridge, England
}

Recent observations of radio emission from Sco X-1 at centimeter wavelengths by Andrew and Purton (1968) and by Ables (1969) have added an important new dimension to the study of galactic X-ray sources. We have considered two alternative interpretations of the radio emission from this object. First, we discuss whether it is possible to attribute the radio emission of Sco X-1 to thermal bremsstrahlung from an optically thin plasma at a temperature of 15 to $20 \mathrm{keV}$. This interpretation would have the merit of relating the radio properties to the existence of a hot, flaring plasma component whose presence has been invoked to explain the observations of hard X-rays ( $\gtrsim 35 \mathrm{keV}$ ) from this object (Peterson and Jacobson, 1966, Buselli et al., 1968, Riegler et al., 1968, Riegler, 1969, Agrawal et al., 1969a, McCracken, 1969). That the $6-\mathrm{cm}$ radio emission and the hard $\mathrm{X}$-radiation should be found to vary by similar amounts over similar time scales lends this hypothesis a certain appeal. However, the measurement at $11 \mathrm{~cm}$ of flux density amounting to $\approx 0.07$ flux units (1 flux unit $\equiv$ $\equiv 10^{-23} \mathrm{erg} \mathrm{cm}^{-2} \mathrm{sec}^{-1} \mathrm{~Hz}^{-1}$ ) reported at this Symposium by McCracken makes it virtually untenable to regard the radio emission of Sco X-1 as arising from thermal origins. Excessive optical and infrared continuum radiation would be produced; moreover, the shape of the radio spectrum is inconsistent with any conceivable thermal model. An explanation for the radio emission of Sco X-1 must therefore be sought in terms of some non-thermal radiation mechanism.

It is realistic to expect that a successful non-thermal model for the radio properties would be only one aspect of a comprehensive treatment of other physical phenomena observed in Sco X-1, such as the hard X-ray flux that is not satisfactorily explained by thermal models. It is also necessary to show how the presence of non-thermal components would relate to and interact with the $5 \mathrm{keV}$ isothermal plasma that is widely believed to produce the observed photon spectra of Sco X-1 from the lowenergy X-ray region to the near infrared by thermal bremsstrahlung.

We have considered a non-thermal model in which the radio flux is attributed to synchrotron-emitting electrons in the relativistic tail of a power-law energy distribution that produces hard X-ray quanta by non-thermal bremsstrahlung. However, the evidence presented earlier at this Symposium by McCracken (1969) and by Agrawal et al. (1969b) for sudden irregular changes in both the intensity and the spectral shape of the hard X-ray component makes it seem presumptive to describe detailed quantitative models at this time. Instead, we should like to restrict our discussion to some of the general properties that might arise from a non-thermal model for the radio emission of Sco X-1. 
The simplest non-thermal model for Sco X-1, in which the radio emission is produced by the electron synchrotron mechanism and the time scale for the radio variability is identified with the characteristic energy-loss time, requires the existence of rather strong magnetic fields *. These might be as intense as $\sim 100 \mathrm{G}$, corresponding to a time scale of $\sim 10^{4} \mathrm{sec}$ for the variations observed at $6 \mathrm{~cm}$ by Ables (1969). There are other possible ways to get significant radio flux changes over a few hours without necessarily invoking the presence of strong magnetic fields, but these alternatives do not have such interesting implications and hence we shall not attempt to consider them at this time.

An intriguing and possibly significant consequence of such a non-thermal model for the radio emission of Sco X-1 is the amount of circular polarization that might be produced by the synchrotron-radiating electrons. For relativistic electrons with a power-law differential energy spectrum and an isotropic velocity distribution emitting synchrotron radiation in a uniform magnetic field, the degree of circular polarization appropriate to the frequency of observation $v$ is given by (Legg and Westfold 1967, 1968)

$$
\Pi_{c}=C(\Gamma)\left[\frac{v_{g} \sin \theta}{v}\right]^{1 / 2} \cot \theta,
$$

which is valid for $\theta \gg \gamma^{-1} \equiv\left(1-\beta^{2}\right)^{1 / 2} \cdot C(\Gamma)$ is a slowly varying function of the electron energy spectral index $\Gamma, v_{\mathrm{g}}$ is the non-relativistic electron gyrofrequency, and $\theta$ is the angle between the magnetic field and the direction of observation. For $C \approx 1, v=2727 \mathrm{MHz}(11 \mathrm{~cm})$, and $B \sim 10^{2} \mathrm{G}$, the degree of circular polarization in the radio emission of Sco $X-1$ could be as high as 20 or $30 \%$. Assuming that we are not situated at a pathological angle with respect to the magnetic field configuration of this source, it seems reasonable to expect $\sim 10 \%$ circular polarization at decimeter wavelengths.

It is worth emphasising that both the amount and the sense of the circular polarization that an observer sees at any particular frequency are mainly determined by the configuration and strength of the magnetic field. The pitch-angle distribution of electron trajectories is relatively ineffective in this regard. Of course, Faraday rotation can have no depolarizing influence on the net degree of circular polarization that the radiation possesses. Only field reversals in the observer's line of sight can reduce the amount of circular polarization estimated for a uniform field with specified orientation.

The possible binary nature of Sco X-1 has been much discussed in recent years following the identification of the X-ray source with a variable optical object that seems to possess some of the characteristics of an ex-nova (Sandage et al., 1966). Attempts have been made by several authors - notably Shklovsky (1967), Cameron and Mock (1967), and Prendergast and Burbidge (1968) - to account for the observed $\mathrm{X}$-ray emission in terms of physical processes appropriate to highly evolved, close binary systems. Although there is as yet no convincing evidence for simple binary motion in Sco X-1, the spectroscopic data are suggestive of complex 'binary' motions

\footnotetext{
* Riegler and Ramaty (1969) seem to have reached a similar conclusion from rather different premises.
} 
possibly combined with gas streams (Westphal et al., 1968). Several speakers at this Symposium have expressed the view that binary models are basically unsupported by observational evidence, but this is far from being a uniformly agreed conclusion. Wilson (1969) has even proposed a new binary model for Cyg X-2, an X-ray object similar to Sco X-1 with an optical counterpart that seems to be a short-period spectroscopic binary (Burbidge et al., 1967; Kristian et al., 1967; but see Kraft and Demoulin, 1967).

The existence of a measurable degree of circular polarization in the radio emission of Sco X-1 might provide a possibility of confirming the essential correctness of such models. A close binary system composed of two stars of approximately one solar mass each, separated by a distance of $\sim 2 \times 10^{12} \mathrm{~cm} *$ would complete an orbit about its centre of mass in about two weeks. If the magnetic field configuration is thus made to change its effective orientation towards the earth, one might expect the degree, and possibly also the sense, of the circularly polarized radio emission to vary regularly with this period. Observation of such an effect would be an important confirmation of the binary-star nature of the Scorpius type of galactic X-ray source.

\section{References}

Ables, J. G.: 1969, Astrophys. J. Letters 155, L27.

Agrawal, P. C., Biswas, S., Gokhale, G. S., Iyengar, V. S., Kunte, P. K., Manchanda, R. K., and Sreekantan, B. V.: 1969a, this volume, pp. 94-106.

Agrawal, P. C., Biswas, S., Gokhale, G. S., Iyengar, V. S., Kunte, P. K., Manchanda, R. K., and Sreekantan, B. V.: 1969b, this volume pp. 94-106.

Andrew, B. H. and Purton, C. R.: 1968, Nature 218, 855.

Burbidge, E. M., Lynds, C. R., and Stockton, A. N.: 1967, Astrophys. J. Letters 150, L95.

Buselli, G., Clancy, M. C., Davison, P. J. N., Edwards, P. J., McCracken, K. G., and Thomas, R. M.: 1968, Nature 219, 1124.

Cameron, A. G. W. and Mock, M.: 1967, Nature 215, 464.

Field, G. B.: 1969, private communication.

Kraft, R. P. and Demoulin, M.-H.: 1967, Astrophys. J. Letters 150, L183.

Kristian, J., Sandage, A. R., and Westphal, J.: 1967, Astrophys. J. Letters 150, L99.

Legg, M. P. C. and Westfold, K. C.: 1967, Proc. Astron. Soc. Australia 1, 27.

Legg, M. P. C. and Westfold, K. C.: 1968, Astrophys. J. 154, 499.

McCracken, K. G.: 1969, this volume, p. 81.

Peterson, L. E. and Jacobson, A. S.: 1966, Astrophys. J. 145, 962.

Prendergast, K. H. and Burbidge, G. R.: 1968, Astrophys. J. Letters 151, L83.

Riegler, G. R.: 1969, Ph.D. thesis, Univ. of Maryland, published as NASA/Goddard Space Flight Center Report X-611-69-1.

Riegler, G. R. and Ramaty, R.: 1969, NASA/Goddard Space Flight Center Report X-611-69-123; 1969, Astrophys. Lett. 4, 27.

Riegler, G. R., Boldt, E. A., and Serlemitsos, P.: 1968, Bull. Am. Phys. Soc. 13, 1434.

Sandage, A. R., Osmer, P., Giacconi, R., Gorenstein, P., Gursky, H., Waters, J., Bradt, H., Garmire, G., Sreekantan, B. V., Oda, M., Osawa, K., and Jugaku, J.: 1966, Astrophys. J. 146, 316.

Shklovsky, I. S.: 1967, Astrophys. J. Letters 148, L1.

Westphal, J. A., Sandage, A., and Kristian, J.: 1968, Astrophys. J. 154, 139.

Wilson, R. E.: 1969, this volume, p. 242.

* This linear dimension is about the minimum that would be consistent with the radio emission becoming optically thick to synchrotron self-absorption at wavelengths longer than $11 \mathrm{~cm}$, assuming $B \sim 100 \mathrm{G}, D \lesssim 100 \mathrm{pc}$ (Field, 1969), and $S_{11 \mathrm{~cm}} \approx 0.07$ flux units. 